\title{
Erratum
}

\section{Vacancy Formation Energy Measurements in Single Crystal Aluminum Using a Variable-Energy Positron Beam}

\author{
K. G. Lynn
}

Physics Department, Brookhaven National Laboratory, Upton, NY 11973, USA

Peter J. Schultz

Physics Department, University of Western Ontario, London, Ontario, Canada N6A 3K7

Appl. Phys. A 37, 31-36 (1985)

In the above paper, Fig. 2 and the analysis described in the text were in disagreement. The original positronium fraction vs. energy data were fit correctly with Eq. (5) where $n$ was allowed to vary as shown in Fig. 3. Figure 2, in the paper, was the case where $n=1$ and not those values shown in Fig. 3 as was implied by (6). If one redoes the analysis with a constant value of $n=1.6$ we find the results shown in the figure

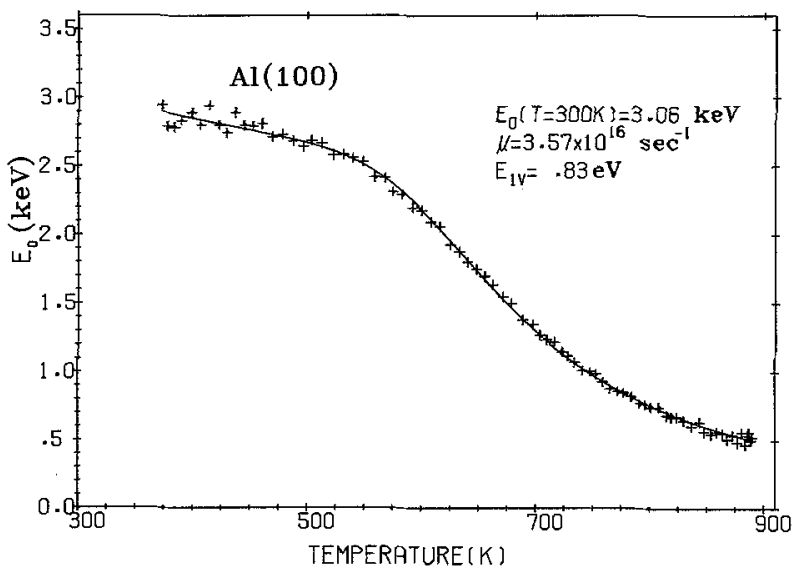

below. The vacancy formation energy of $0.83 \mathrm{eV}$ and a high specific trapping rate, $\mu$, is in disagreement with the accepted formation enthalpies measured in bulk $\mathrm{Al}$ by other techniques $(0.6-0.7 \mathrm{eV})[1,2]$. If the value of $n$, as shown in Fig. 3 of the original manuscript, is used a formation energy $>.9 \mathrm{eV}$ is extracted. At the present time we do not completely understand this discrepancy. A more thorough explanation will be discussed in a later publication, however it should be emphasized that until this discrepancy is resolved the slow positron technique does not appear to provide reliable formation enthalpies.

Acknowledgement. We wish to thank A. Vehanen for making us aware of this discrepancy.

\section{References}

1. M.J. Fluss, L.C. Smedskjaer, M.K. Chason, D.G. Legnini, R.W. Siegel: Phys. Rev. B 17, 3444 (1978)

2. H.E. Schaefer, R. Gugelmeier, M. Schmolz, A. Seeger: Proc. of the $5^{\text {th }}$ Ris $\phi$ International Symposium on Metallurgy and Materials Sciences (to be published) 